\title{
Development of biliary sludge in patients on intensive care unit: results of a prospective ultrasonographic study
}

\author{
F E Murray, S J Stinchcombe, C J Hawkey
}

\begin{abstract}
Biliary sludge may be a precursor of gall stones in man. The aim of this study was to determine the incidence of biliary sludge in a prospective study of 36 patients admitted to the intensive care unit for longer than two days. The presence of biliary sludge was determined by ultrasonography. Biliary sludge developed in 17 patients (47\%), after a mean of 5.5 days in the intensive care unit. Patients who developed biliary sludge spent longer in the intensive care unit (14.2 d (1.3)), compared with patients who did not $(8.3 \mathrm{~d}(1.4)) ;(p=0.003)$. Ten of the patients with biliary sludge had a recognised risk factor: total parenteral nutrition (five), abdominal surgery (two), or both (three). All neurosurgical patients (four) who required total parenteral nutrition developed biliary sludge. Seven patients with biliary sludge had no previously recognised risk factor, five of whom had severe head trauma or neurosurgery. In conclusion, biliary sludge develops frequently and rapidly in patients admitted to an intensive care unit. Neurosurgical procedures are associated with biliary sludge formation. (Sludge is commonly associated with the development of cholestatic liver biochemistry.) (Gut 1992; 33: 1123-1125)
\end{abstract}

Biliary sludge is recognised ultrasonographically as echogenic material which layers in the most dependent portion of the gall bladder and does not produce acoustic shadowing. ${ }^{1}$ The major constituents are supersaturated bile, mucin glycoprotein in high concentrations and unconjugated bilirubin. ${ }^{2}$ A large body of experimental and clinical evidence suggests that biliary sludge is a precursor of gall stones in a large number of affected patients. ${ }^{3}$ Several risk factors have been identified. They include total parenteral nutrition, ${ }^{4}$ post bone marrow transplantation, ${ }^{5}$ pregnancy, ${ }^{6}$ AIDS, ${ }^{7}$ and after abdominal surgery.$^{8}$ In many subjects, however, there is no clear risk factor.

Uncontrolled clinical impressions have led

Departments of

Therapeutics and

Radiology, University

Hospital, Queens

Medical Centre,

Nottingham

F E Murray

S J Stinchcombe

C J Hawkey

Correspondence to:

Dr F E Murray, University Department of Therapeutics, University Hospital, Queens Medical Centre, Nottingham NG7 2UH.

Accepted for publication

11 November 1991

\section{Methods}

PATIENTS

Thirty six patients admitted to the intensive care unit at the University Hospital, Queens Medical Centre, Nottingham, for more than two days over an 11 week period were investigated. Biliary ultrasonography was performed by the same investigator (SJS), using an Aloka SSD-650 real time ultrasound machine with $5 \mathrm{mHz}$ and $3.5 \mathrm{mHz}$ curved linear array transducers. Biliary sludge was recognised as echogenic material which layered in the most dependent portion of the gall bladder without acoustic shadowing (Fig 1), but was also recognised as poorly defined globular masses of low echogenicity within the gall bladder (Fig 2). Initially these masses were not recognised as sludge, although they were of the same echogenicity. In these patients, however, subsequent scans always showed the typical layered appearance of biliary sludge. It is likely that in certain patients sludge initially forms as a globular mass which subsequently layers into the most dependent part of the gall bladder. Scans were done twice a week on average. Statistical analysis was performed using Student's unpaired $t$ test and Fisher's exact test.

All patients except two were mechanically ventilated. The male to female ratio was $26: 10$ with an average age of 47 years (range 17-80). Underlying major illnesses included neurosurgery/severe head injury 19 , multiple trauma six, abdominal surgery eight (vascular surgery five, gastrointestinal surgery three), others (Gram negative sepsis, epiglottitis, encephalitis/ post cardiac arrest) three. Three patients had major bacterial sepsis on admission to the intensive care unit.

Ten patients received total parenteral nutrition early in their treatment in the intensive care unit, and three at a later stage. On average, total parenteral nutrition began $3 \cdot 1$ days after admission to the intensive care unit (range 0-7).

\section{Results}

Biliary sludge developed in 17 of 36 patients (47\%), after mean 5.5 days (SEM 0.9 ). Three of these patients had sludge on their initial scan (one patient who had undergone aortic aneurysm repair five days previously; one had a subarachnoid haemorrhage and subsequent repair of intracranial aneurysm in the previous week; one had undergone intracranial haemorrhage three days before). Subjects who developed biliary sludge spent longer in the intensive care unit $14 \cdot 2$ (SEM 1.3) days, than those who did not $8 \cdot 3$ (SEM 1.4) days $(\mathrm{p}=0.003)$.

In seven biliary sludge forming patients, there was no previously recognised risk factor. Of those seven, five had undergone neurosurgery or had received a severe head injury (having 
Figure 1: Ultrasonographic appearance of biliary sludge showing echogenic

shadowing which layers in

the most dependent portion of

the gall bladder, without acoustic shadowing.

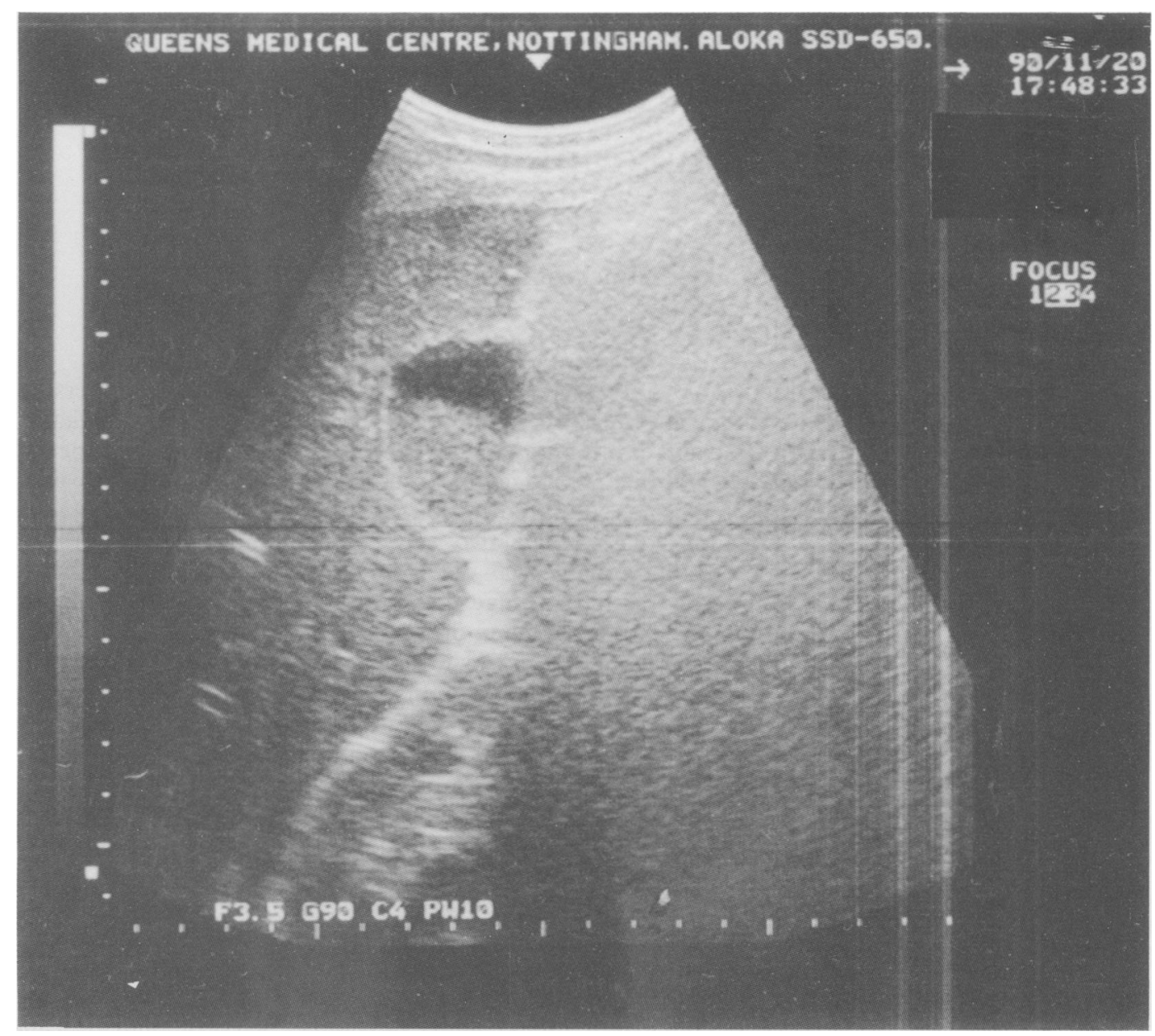

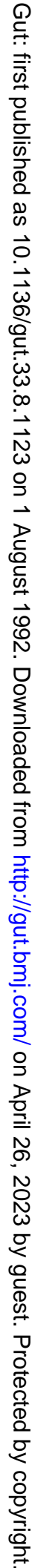

QUEENS MEDICAL CENTRE, NOTTIMGHAM. ALOKA SSD-650.

$\rightarrow \quad 90 / 10 / 30$

FOCus

1234
Figure 2: Ultrasonographic appearance of biliary sludge showing echogenic

shadowing in the most

dependent portion of the gall bladder, without acoustic shadowing, but with a

globular appearance to the superior surface. 
Frequency of development of biliary sludge in patients admitted to the intensive care unit for longer than two days

\begin{tabular}{lcccc}
\hline & Total & Sludge & $\begin{array}{l}\text { No } \\
\text { sludge }\end{array}$ & $\begin{array}{l}\% \\
\text { sludge }\end{array}$ \\
\hline Total parenteral nutrition & 2 & 1 & 1 & 50 \\
Total parenteral nutrition plus & & 3 & 1 & 75 \\
$\quad$ abdominal surgery & 4 & 3 & 1 & \\
Total parenteral nutrition plus & & & 0 & 100 \\
$\quad$ neurosurgery/head injury & 4 & 4 & 2 & 50 \\
Abdominal surgery & 4 & 2 & 10 & 33 \\
Neurosurgery/head injury & 15 & 5 & 5 & 29 \\
Various & 7 & 2 & 19 & 47 \\
Total & 36 & 17 & 19 & \\
\hline
\end{tabular}

received enteral nutrition but no total parenteral nutrition), one had multiple trauma, and one Gram negative septicaemia. Ten of the biliary sludge forming patients had a previously recognised risk factor: total parenteral nutrition (five), abdominal surgery (two) or both abdominal surgery and total parenteral nutrition (three). Thus, five of eight subjects who underwent abdominal surgery developed sludge, as did eight of 10 subjects receiving total parenteral nutrition. All neurosurgical patients who received total parenteral nutrition (four) developed biliary sludge. Thus nine of 19 patients who underwent neurosurgery or had a severe head injury developed biliary sludge. The development of biliary sludge in patients receiving total parenteral nutrition was more rapid than previously recognised in patients receiving total parenteral nutrition outside the intensive care unit (mean $3 \cdot 1$ days). The frequency of development of biliary sludge in the different patient groups is shown in the Table.

Liver biochemistry was performed regularly in 33 of 36 patients. Cholestasis developed in five of 16 non-sludge forming subjects and in 13 of 17 sludge forming subjects; this difference is statistically significant $(p=0 \cdot 01)$. None of the patients in this series developed evidence of acute acalculus cholecystitis, and thus the development of abnormal liver biochemistry does not appear to relate to the development of this complication.

APACHE scores, an index of illness severity and prognosis widely used in patients on the intensive care unit, were obtained on admission to the intensive care unit in all subjects except two, and were similar in the non-sludge and sludge forming groups: non-sludge forming mean APACHE score 11.8 (SEM 1.9), sludge forming $14 \cdot 1$ (SEM 1.7); $\mathrm{p}=0 \cdot 38$.

\section{Discussion}

The aim of this study was to evaluate the incidence of biliary sludge in critically ill patients admitted to the intensive care unit for more than two days. The major findings of this study are that biliary sludge develops frequently and rapidly in intensive care patients, and that the spectrum of patients who develop sludge is wider than previously recognised. In particular, we recognise major neurosurgical procedures and severe head injury as a risk factor for biliary sludge development, developing in nine of 19 subjects.
The classic ultrasonographic appearance of biliary sludge is that of echogenic material which layers in the most dependent portion of the gall bladder.' In some of our subjects, however, a globular mass was initially seen in the gall bladder (Fig 2). We did not initially recognise this as biliary sludge, but believe that it represents an alternative appearance of biliary sludge, reflecting the high mucin glycoprotein content and thus high viscosity of biliary sludge ${ }^{2}$ and is analogous to the globular appearance noted by Lee et $a l$ in the in vitro sheep model of biliary sludge. ${ }^{2}$

Because gall stone formation is a frequent result of biliary sludge formation in all groups previously examined, this study may have potential therapeutic implications. Further research may focus on the subsequent incidence of gall stones and on developing a regime to prevent or clear biliary sludge in at risk patients. Potential candidates for such research include daily intravenous infusions of CCK-OP (cholecystokinin). ${ }^{9}$ CCK-OP has been shown to reduce biliary sludge formation in patients receiving total parenteral nutrition. ${ }^{9}$ Other potential candidates include agents which inhibit prostaglandin synthesis, because prostaglandins may be important in mediating secretion of gall bladder mucin glycoprotein, an essential component of biliary sludge and subsequent gall stone formation. ${ }^{10}$ Finally rapid intermittent intravenous amino acid infusions have recently been shown to stimulate gall bladder contractility, and may provide an acceptable method of preventing biliary sludge in patients receiving total parenteral nutrition. ${ }^{1 "}$

This work was presented at the Medical Research Society meeting Cambridge, UK in July 1991, and was published in Clinical Science in abstract form (Clin Sci 1991; 81: 26). We wish to thank the anaesthetists, surgeons, and physicians in University Hospital for permission to study their patients, and the Sister and nursing staff of the intensive care unit for their help in performing this study.

1 Conrad MR, Jones RO, Dietchy J. Significance of low level echoes in the gallbladder. Am $\mathcal{F}$ Roentgenol 1979; 132: $967-72$

2 Lee SP, Nicholls JF. Nature and composition of biliary sludge. Gastroenterology 1986; 90: 677-86.

3 Lee SP, Maher K, Nicholls JF. Origin and fate of biliary sludge. Gastroenterology 1988; 94: 170-6.

4 Messing B, Bories C, Kunstlinger F, Bernier JJ. Does total parenteral nutrition induce gallbladder sludge formation and lithiasis. Gastroenterology 1983; 84: 1012-9.

5 Frick MP, Snover DC, Feinberg SB, Salomonowitz E, Crass JR, Ramsay NKC. Sonography of the gallbladder in bone marrow transplant patients. Am $\mathcal{F}$ Gastroenterol 1984; 79: marrow $122-7$.

6 Maringhini A, Marceno MP, Lanzarone F, Caltagirone M, Fusco G, Di Cuonzo G, et al. Sludge and stones in gallbladder after pregnancy. $\mathcal{f}$ Hepatol 1987; 5: 218-22.

7 Grumbach K, Coleman BG, Gal AA, Arger PH, Mintz MC, Arenson $\mathrm{RL}$, et al. Hepatic and biliary tract abnormalities in patients with AIDS. F Ultrasound Med 1989; 8: 247-54.

8 Bolondi L, Gaini S, Testa S, Labo G. Gallbladder sludge formation during prolonged fasting after gastrointestinal tract surgery. Gut 1985; 26: 734-8.

9 Sitzmann JV, Pitt HA, Steinborn PA, Pasha ZR, Sanders RC. Cholecystokinin prevents parenteral nutrition induced biliary sludge in humans. Surg Gynecol Obstet 1990; 170: 25-31.

10 Lee SP, Carey MC, LaMont JT. Aspirin prevention of cholesterol gallstone formation in the prairie dog. Science 1981; 211: 1429-31.

11 Nealon WH, Upp JR, Alexander RW, Gomez G, Townsend $\mathrm{CM}$, Thompson JC. Intravenous amino acid acids stimulate human gallbladder emptying and hormone release. Am F Physiol 1990; 259: G173-8. 